\title{
Le connétable d'Eu et son cercle nobiliaire
}

Le réseau de familiers d'un grand seigneur au $\mathrm{XIV}^{\mathrm{e}}$ siècle

\section{Émilie Lebailly}

\section{(2) OpenEdition}

Journals

Édition électronique

URL : https://journals.openedition.org/crm/747

DOI : $10.4000 / \mathrm{crm} .747$

ISSN : 1955-2424

Éditeur

Honoré Champion

Édition imprimée

Date de publication : 30 décembre 2006

Pagination : 41-52

ISSN : 1272-9752

Référence électronique

Émilie Lebailly, «Le connétable d'Eu et son cercle nobiliaire », Cahiers de recherches médiévales [En ligne], 13 | 2006, mis en ligne le 27 novembre 2009, consulté le 15 décembre 2022. URL : http:// journals.openedition.org/crm/747 ; DOI : https://doi.org/10.4000/crm.747 


\section{ris}

\section{Le connétable d'Eu et son cercle nobiliaire : le réseau de familiers d'un grand seigneur au XIV $\mathrm{XV}^{\mathrm{e}}$ siècle}

Les pages qui suivent condensent les éléments d'une thèse de doctorat consacrée à Raoul, comte d'Eu, connétable de France, à sa vie, et au cercle de chevaliers qui gravitaient autour de lui. Nous présentons ici de façon synthétique les conclusions d'une étude prosopographique menée sur son entourage noble afin de mettre en lumière le système de clientèle et de réseau à la base de nombre de relations humaines au sein de la noblesse du royaume de France dans la première moitié du $\mathrm{XIV}^{\mathrm{e}}$ siècle. Mais il convient d'abord de présenter succinctement le chef (au sens étymologique du terme) de ce réseau, le comte d'Eu, connétable du roi Philippe VI de Valois, sa vie et son action militaire pendant les premières campagnes de la guerre de Cent ans. Nous définirons ensuite les éléments constitutifs de son réseau avant d'analyser le choix des hommes, les liens qui les unissaient au connétable pour finir par la destinée de ce réseau après la disparition du connétable d'Eu.

Raoul, comte d'Eu et de Guînes, a occupé l'office de connétable de France sous le règne de Philippe VI de Valois. Né dans les années 1290, il était contemporain de ce roi et des fils de Philippe le Bel. Ses parents appartenaient à la haute noblesse du royaume de France : son père, Jean de Brienne, comte d'Eu, représentait la branche cadette de l'illustre famille champenoise des Brienne, qui furent rois de Jérusalem au XIII ${ }^{\mathrm{e}}$ siècle, et descendait de la maison de Lusignan; sa mère, Jeanne de Guînes, héritière de ce comté qu'elle transmit à son fils, descendait pour sa part de la maison de Coucy. Le connétable d'Eu pouvait ainsi s'enorgueillir d'une illustre ascendance comme d'enviables alliances matrimoniales puisqu'il était apparenté à un grand nombre des familles nobles de son temps, comme les maisons de Châtillon Saint-Pol ou encore de Noyers. Il avait épousé Jeanne de Mello, une riche héritière bourguignonne et était l'un des barons français les mieux possessionnés du royaume : par son mariage et par héritage paternel ou maternel, il possédait en effet des terres dans plusieurs régions comme la Normandie, la Picardie, la Bourgogne ou encore la Saintonge ; son cercle d'influence était donc loin de se limiter aux comtés d'Eu et de Guînes qu'il tenait de ses parents.

Sa famille paternelle faisait partie de l'entourage royal depuis plusieurs générations : son arrière-grand-père et son arrière grand-oncle avaient été bouteiller et chambellan de Saint Louis, son père et son grand-père étaient des familiers de la cour royale. Serait-ce cette tradition du «service public» dans sa famille qui aurait présidé à son choix pour succéder au connétable Gaucher de Châtillon après sa mort en 1329 ?

Raoul d'Eu devint en effet connétable de France en 1329, succédant à Gaucher de Châtillon, connétable depuis 1302, après la bataille de Courtrai. Le comte d'Eu avait bien connu son prédécesseur: il avait servi dans son entourage à ses débuts, notamment en $1320^{1}$. Quatre ans plus tard, il était chargé de surveiller les côtes

${ }^{1}$ Chronographia Regum Francorum, éd. H. Moranvillé, Paris, 1893, t. 1, p. 247.

Cahiers de Recherches Médiévales, 13, 2006 
françaises de Leure à Calais, conformément aux ordres de Gaucher ${ }^{2}$. Leurs deux familles étaient par ailleurs unies par des mariages, ce qui laisse supposer, étant donné la différence d'âge entre les deux hommes, que Gaucher fut en quelque sorte le parrain d'armes du comte d'Eu. Cette impression est renforcée par le fait qu'il servait sous ses ordres la première fois qu'il apparut dans un fait d'armes. De plus, les parents du comte d'Eu étant des familiers de l'entourage royal, tout comme Gaucher de Châtillon, il est aisé d'imaginer qu'ils s'étaient largement fréquentés à la cour de Philippe le Bel et que le comte d'Eu fut, en son temps, recommandé au connétable pour ses premières armes. Il a très probablement gravité dans son cercle. Cette relation fut sans aucun doute un atout supplémentaire qui lui permit d'être considéré comme le meilleur candidat à l'un des offices les plus enviés du royaume. Cet épisode permet déjà de percevoir l'importance des réseaux de «familiers » au sein de la noblesse de cour du XIV siècle. $^{\mathrm{e}}$

En tant que connétable de France, il assumait par définition le rôle de conseiller militaire du roi, à côté des deux maréchaux de France et du maître des arbalétriers, mais pas seulement : membre du conseil royal, il pouvait conseiller le roi dans tous les domaines, à l'instar des autres membres du conseil, qui étaient alors des hommes polyvalents s'occupant des diverses affaires du royaume, intérieures et extérieures. Ainsi le connétable Gaucher de Châtillon fut-il l'ambassadeur privilégié de Philippe IV en de nombreuses occasions, chargé de plusieurs missions diplomatiques qu'il remplit avec succès.

En 1340, Raoul d'Eu présenta au roi diverses requêtes, fondées ou infondées, en vue de son profit, tant ses besoins financiers étaient élevés ${ }^{3}$. Pour simplifier, il demandait qu'étant donné ses responsabilités, soit comme connétable de France, le roi présent ou bien le lieutenant du roi, soit, le cas échéant, comme lieutenant du roi en personne (ce qui lui arrivait), à être non point aux gages du roi (car, selon lui les gages étaient loin de couvrir les frais, et notamment la perte des chevaux d'armes) mais aux «frais, cousts et restors » du roi, autrement dit aux frais réels, pour lui et son «hôtel et ménage »; il demandait en outre à prélever son droit de connétable (une journée de gages) sur tous les gens d'armes et de pied à la solde du roi, qu'ils viennent d'au-delà du royaume (tels le roi de Bohême, les évêques de Liège et de Metz, le comte de Savoie) ou qu'ils soient sous l'autorité des membres du lignage royal (tels le roi de Navarre, le comte d'Alençon et le duc de Bourgogne); il ne pouvait en revanche rien demander sur les gens de guerre servant à leurs dépens (ce qui semble logique) ni sur les «soudoyers de la mer» dont les droits appartenaient à l'amiral et au clerc des arbalétriers; il demandait également 100 livres tournois par jour quand le roi chevauchait «armé, bassinet en teste» et seulement 50 livres tournois quand il chevauchait sans bassinet, plus un cheval «pour monter après l'oriflambe ». Il demandait encore, quand une ville était prise «a force», l'ensemble du butin, sauf les prisonniers et l'artillerie. Tout cela pouvait paraître exorbitant, mais il affirmait que sans cette généreuse prise en charge par le trésor royal, sans ses diverses indemnités, il lui serait impossible de recruter les 40-50 bannerets et bacheliers et les 40-50 écuyers nécessaires à son hôtel (retenons ce chiffre), et il serait obligé de vendre la plus grande partie de ses terres, et encore cela ne suffirait pas.

${ }^{2}$ B.n.F., fr. 9501, f. $94 \mathrm{r}^{\circ}$

${ }^{3}$ A.N., JJ 269, f. $86 \mathrm{r}^{\circ}-87 \mathrm{v}^{\circ}$. 
L'intérêt de ces demandes est aussi de préciser son rôle en tant que connétable : il lui appartenait, quand le roi chevauchait en bataille, de visiter toutes les batailles, de voir leur arroi et leur «conroi »; quand il s'arrêtait (il était statutairement à l'avantgarde), toute l'armée devait s'arrêter, selon un signal transmis aux maréchaux de France; en revanche, s'il chevauchait, toute l'armée devait chevaucher; une fois les lignes de bataille disposées par ses soins, nul ne pouvait bouger de sa place avant qu'il ne tourne le dos aux lignes de bataille, «le visage a l'ennemi » et l'ost royal derrière lui. Bref, assisté des maréchaux, un connétable de France avait comme principale tâche, sur le terrain, de mettre un peu d'ordre et de discipline au sein de la cohue de nobles et de communes que représentait à cette date l'ost royal.

La guerre de Cent ans ayant débuté en 1337, le comte d'Eu fut envoyé par Philippe VI sur les différents fronts : en Languedoc en été 1337 puis dans le nord du royaume de 1338 à 1340 . On sait que les toutes premières années de ce long conflit n'ont pas été déterminantes, malgré la disparité des forces en présence. En 1339 puis en 1340, des «batailles assignées » furent envisagées entre le roi de France et son adversaire - et compétiteur - Édouard III mais elles furent pour ainsi dire décommandées au dernier moment.

La première grande bataille terrestre de la guerre de Cent ans fut celle de Crécy, le 26 août 1346. Or le connétable d'Eu mourut le 19 janvier 1345, d'un coup de lance dans le ventre lors de joutes données à l'occasion des noces du fils cadet du roi, le duc d'Orléans. Seul exemple d'hérédité de cet office majeur, son fils lui succéda en tant que connétable durant les cinq dernières années du règne de Philippe VI. Prisonnier des Anglais au siège de Caen dès 1346, il passa plusieurs années en captivité à Londres avant d'être libéré. À peine arrivé sur le sol français pour réunir sa rançon, il était arrêté, jugé et décapité pour trahison (on aurait retrouvé sur lui des lettres d'allégeance au roi d'Angleterre Édouard III) par ordre du nouveau roi, Jean, le 19 novembre 1350.

Revenons à Raoul I. On ne peut attribuer de beaux faits d'armes au premier connétable, du fait des circonstances mêmes dans lesquelles se déroulèrent les premières campagnes de la guerre: sièges, escarmouches, attaques, reculs, refus de l'engagement et indécision, imputables au souverain en premier lieu, à son conseil au sens large ensuite. L'intérêt d'étudier ce personnage ne résidait donc pas dans sa vie ni dans sa carrière militaire, mais bel et bien dans le cercle de familiers dont il a su s'entourer lors des campagnes auxquelles il prit part. En effet, la nécessité d'étudier son réseau se révéla à la lecture des sources militaires du temps : la présence assidue sur plusieurs années des même hommes dans ses montres et ses batailles laissait supposer qu'il avait choisi ou «retenu» (selon l'expression du temps) les mêmes hommes pendant la plupart des campagnes du début de la guerre de Cent ans. Il fallait dès lors se pencher sur ce réseau d'hommes d'armes au service du connétable : comment et quand fut-il constitué, comment fonctionnait-il, qui étaient ces hommes ? Combien étaient-ils ? En d'autres termes, comment le comte d'Eu les avait-t-il recrutés et pourquoi ?

Deux types de documents concernant les «états de service» du connétable d'Eu sont parvenus jusqu'à nous : tout d'abord son registre de comptes ${ }^{4}$, puis les

${ }^{4}$ A.N., JJ 269, en cours de publication. 
retenues de son hôtel et ses montres d'armes ${ }^{5}$. Or, tout au long des années 13371340, nous retrouvons dans ces deux sources les mêmes individus. Ces sources permettent de repérer un noyau de fidèles chevaliers entourant le comte lors des campagnes. Il était dès lors intéressant d'étudier sa constitution, le choix de ses membres, les raisons de ce choix, de même que la carrière de ces hommes après la mort du connétable car la plupart d'entre eux lui survécurent plus de dix ans. Après un recensement des chevaliers servant à l'hôtel et/ou sous la bannière du comte d'Eu, il ressort qu'une cinquantaine d'individus l'accompagnèrent tout au long de ces quelques années, la moitié appartenant à son hôtel et paraissant réellement proche de lui, les autres étant plutôt des «familiers d'armes ».

Une fois les chevaliers sélectionnés, l'étude du réseau et de sa constitution pouvait commencer. La question primordiale pour comprendre le fonctionnement du cercle de familiers du connétable nous sembla être de définir la nature du lien qui unissait le comte à ses hommes, avant l'origine géographique, bien que cette dernière soit déjà un indice du choix opéré par le comte d'Eu. Passons donc en revue rapidement les origines des familiers du connétable, qu'ils soient de l'hôtel ou non. Le principal fief du connétable étant situé aux frontières de la Normandie et de la Picardie, il n'est pas surprenant de rencontrer une écrasante majorité de barons normands et picards dans son entourage ; ainsi sur les 28 familiers de l'hôtel, la moitié sont normands et cinq viennent de Picardie, tandis que sur les 26 «familiers d'armes » on dénombre neuf Normands, trois Picards, trois chevaliers du nord du royaume, trois Bourguignons, un Barrois, un vassal du comté d'Eu, un Saintongeais et quatre parents du comte. On voit dores et déjà se dessiner le mode de recrutement du connétable et par conséquent la nature du lien l'unissant à ses hommes.

Ayant établi la diversité d'origine de l'entourage du connétable, nous allons entrer au cœur de notre problématique et tenter de définir de façon précise la nature du lien qui unissait le comte d'Eu et ses chevaliers, car ce lien est souvent beaucoup plus complexe qu'il n'y paraît et ne revêt rarement qu'un seul aspect d'une relation à plusieurs niveaux. Et c'est ainsi qu'il sera possible de saisir la constitution et le fonctionnement du réseau de familiers du comte d'Eu.

À son échelle, l'entourage de chevaliers du comte d'Eu offre à l'historien un fidèle reflet de la société militaire du $\mathrm{XIV}^{\mathrm{e}}$ siècle. En ce sens, les quatre grandes formes de solidarités traditionnellement rencontrées dans les armées, et mises en avant par Philippe Contamine ${ }^{6}$, apparaissent largement dans cette étude : solidarité régionale, lignagère, vassalique et enfin les retainers (les chevaliers dont le comte paie les gages et qui sont mentionnés dans son registre de dettes). Nous verrons que ce dernier lien vient compléter la plupart des autres. Puisqu'elles reflètent la façon dont le connétable «retenait» ses hommes, reprenons une à une ces attaches : le lien régional, le lien vassalique et le lien familial et lignager, avant de constater qu'ils sont tous liés et s'interpénètrent étroitement.

\footnotetext{
${ }^{5}$ Principalement B.n.F., fr. 9501, fr. 20685 et fr. 32510. Il faut ajouter le compte de Barthélemy du Drach, trésorier des guerres de Philippe VI, de la bataille de Raoul, comte d'Eu, connétable de France, lieutenant du roi sur les frontières de Flandre et de Hainaut, allant du 9 mars 1340 au $1^{\text {er }}$ octobre 1340 (B.n.F., n.a.fr. 9238, f. $127 \mathrm{v}^{\circ}-147 \mathrm{r}^{\circ}$ ).

${ }^{6} \mathrm{Ph}$. Contamine, Guerre, État et société à la fin du Moyen Âge. Étude sur les armées de rois de France (1337-1494), Paris-La Haye, 1972, p. 604-605.
} 


\section{La solidarité régionale}

Il s'agit ici, et de très loin, du mode de recrutement préféré du comte d'Eu. En effet, plus de la moitié des chevaliers de son entourage, qu'ils servent dans l'hôtel ou aux armées, étaient des voisins proches ou lointains, et la quasi totalité était originaire du quart nord-ouest du royaume. Cela rejoint l'idée que Raymond Cazelles avait émise sur la communauté des pays de l'Ouest, mais il est cependant possible d'affiner ce concept dans la mesure où la plupart de ces voisins étaient normands ou picards. Les liens unissant ces deux provinces ne sont plus à démontrer, qu'il s'agisse de la langue, de la littérature ou encore du mode de pensée.

Les voisins normands du comte d'Eu figuraient au nombre de vingt-deux : Pierre de Bailleul, Guillaume et Jean de Beuzeville, Guillaume de Bréauté, Jean Doublel, Robert de Houdetot, Guillaume, Jean et Robert de La Heuse, Jean de Malvoisin, Robert et Thomas de Marigny, Philippe de Pons, Pierre de Préaux, Robert de Thiois et Jean Valiquet. Ces hommes étaient tous des nobles chevaliers issus d'un certain milieu, mais il convient de leur ajouter quelques voisins de plus modeste extraction, comme Colin Doublel et les cinq membres de la famille de Hotot, fidèles serviteurs de l'hôtel du comte.

Après cette forte présence des barons normands, les compagnons du comte d'Eu venaient assez massivement du nord du royaume, principalement de la Picardie, et plus particulièrement de l'actuel département de la Somme. On dénombre treize barons picards : Jean de Bailleul, Jean de Bauchain, Jean de Cayeu, Matthieu de Cayeu, Jean de Dargnies, Jacques d'Estréelles, Jean de Fricamps dit Friquet, Guillaume du Quesnoy, Jean de Maisy, Enguerrand Quiéret, Guillaume de Villiers et Jean de Walaincourt.

Normands et Picards formaient la majorité des retenues du connétable: on constate donc combien joue ici, à plein, la solidarité régionale. Elle est par ailleurs perceptible dans d'autres régions et s'apparenterait alors à la solidarité entre vassaux d'un même seigneur. En effet, les autres voisins territoriaux du comte qui servirent sous sa bannière étaient des barons bourguignons et barrois. Les voisins bourguignons étaient à la fois peu nombreux et peu assidus dans la compagnie du connétable. Ils étaient au nombre de quatre: Geoffroy de Charny, Édouard de Beaujeu, Eudes de Grancey, et Guillaume de Bordeaux. La faible assiduité des deux plus grands seigneurs, Édouard de Beaujeu et Geoffroy de Charny, peut s'expliquer par leur rang même puisqu'ils étaient, tout comme le connétable, des proches de l'entourage royal et se trouvaient investis, comme lui, de commandement lors des campagnes militaires. S'ils ont peu combattu et servi le roi ensemble, ils le firent en même temps, avec chacun leur commission de lieutenant ou de capitaine émanant du souverain, mais pas nécessairement au même endroit. Il reste enfin à évoquer deux chevaliers originaires du comté de Bar, Louis de Courbon et Ferry de Chardogne. Qu'il s'agisse des Bourguignons ou des Barrois, ces chevaliers possédaient des terres voisines de celles du comte d'Eu et leur particularité est qu'ils étaient, si l'on peut dire, des compagnons de vassalité du comte, ou des co-vassaux. En effet, le connétable détenait des terres en Bourgogne, dans l'Auxerrois et le Nivernais, et d'autres qu'il tenait en fief du comte de Bar, du chef de sa femme, comme les châteaux et les villages sur l'Yonne entre Cravant et Auxerre. Il semblerait donc qu'une certaine solidarité soit de mise entre les vassaux d'un même seigneur, lesquels n'hésitaient pas à servir sous le gouvernement de l'un ou l'autre. Ainsi, même si les 
possessions du comte d'Eu n'étaient pas directement proches de celles des chevaliers barrois Ferry de Chardogne ou Louis de Courbon, le lien régional opérait tout de même.

De la sorte, le lien de voisinage pouvait revêtir différentes formes, proches ou lointaines, comme différents degrés, directs ou indirects, dans le sens où les grands seigneurs indépendants et voisins s'entraidaient mais cette assistance jouait également pour les chevaliers vassaux du même seigneur que le connétable. On voit donc que le comte d'Eu avait réussi à rallier beaucoup de nobles du duché de Normandie sous sa bannière, mais également des Picards, des Bourguignons et des Barrois. Il a su susciter des vocations, des fidélités qui ne se démentirent pas de son vivant et qui continuèrent d'opérer bien après sa mort, jusque dans les rangs des fidèles du roi de Navarre ou du duc de Normandie.

\section{La solidarité vassalique}

Bien que son importance dans la société féodale ne soit plus à démontrer, le lien d'homme à homme n'était pas un mode de recrutement usité dans la compagnie du connétable : peu de vassaux l'accompagnèrent dans ses campagnes.

Les chevaliers qui étaient ses vassaux venaient exclusivement de ses deux terres patrimoniales principales, le comté d'Eu et le comté de Guînes. Ce dernier compte comme la deuxième terre du connétable par ordre de taille et d'importance géographique et économique. Il aurait toutefois pu prétendre à la première place géopolitique car ce territoire était largement convoité par Édouard III qui pensait en faire un port de débarquement aisé pour ses troupes (c'eût été un bon moyen, par ailleurs, d'agrandir son petit domaine calaisien après 1347).

Les vassaux du connétable étaient au nombre de cinq : Baudouin et Tassart de Balinghem, originaires de la petite ville éponyme, toute proche de Guînes, et Geoffroy du Forestel, Frémaut d'Oust et Anguenier de Sainte-Beuve, du comté d'Eu. Par ce lien, ils s'étaient faits les hommes du connétable au premier chef et pour cette raison, ils lui devaient le service armé. Les deux hommes du Boulonnais sont cités dans le registre de comptes du comte, ainsi que Geoffroy du Forestel, mais on n'y rencontre pas les deux autres Normands.

Ces petits nobles étaient probablement les plus dépendants de leur seigneur, dans le sens où ils étaient ses hommes au premier chef, puisque leur seigneurie était située au cœur des deux comtés. Ils étaient sans doute les obligés du comte, peut-être même vivaient-ils en partie grâce à ses largesses. L'assiduité d'un chevalier comme Anguenier de Sainte-Beuve à accompagner le connétable pourrait s'expliquerait comme cela. Est-ce pertinent de qualifier ce comportement d'opportunisme ? Car ce fut sans aucun doute une chance pour un chevalier de la petite noblesse, titulaire d'un modeste fief, de voir son seigneur accéder à l'office de connétable de France, l'un des plus importants du royaume. Dès lors, devenir l'un de ses proches compagnons d'armes et graviter dans son entourage, le servir assidûment, ne pouvait que rendre sa vie plus aisée. On réalise en effet, à la lecture du registre de dettes du connétable, qu'à ses yeux, la largesse et la prodigalité n'étaient pas un vain mot.

Il faut toutefois relever l'absence de chevaliers poitevins et saintongeais, voisins ou vassaux du comte d'Eu, l'exception étant incarnée par Alain de Montendre, originaire de Saint-Jean-d'Angély. Cette absence reflèterait-elle le peu d'importance 
que le comte aurait accordé à ses terres du Poitou ? Aussi nombreux que ses vassaux, les parents du connétable trouvèrent également une place dans son entourage.

\section{La solidarité familiale et lignagère}

Le troisième lien qui unissait ces hommes au comte d'Eu est d'ordre familial. Mais il est significatif de constater que les quatre parents du connétable figurant dans les sources n'appartinrent pas à sa maison mais le servirent uniquement en campagne: il ne choisissait pas ses intimes au sein de sa famille, mais plus volontiers parmi les barons normands, comme nous l'avons vu plus haut.

À tout seigneur tout honneur, son fils Raoul, comte de Guînes, l'accompagna dans la totalité de ses campagnes. Viennent ensuite les cousins de son épouse, Guillaume de Mello, seigneur d'Époisses, et Dreu, seigneur de Saint-Bris, implantés en Bourgogne. Ce sont les seuls parents proches qui le suivirent dans toutes ses campagnes. Figurant dans huit retenues, on peut sans conteste les qualifier de familiers d'armes du comte d'Eu. Ils constituaient ainsi le noyau dur de son entourage militaire, sa garde rapprochée qui le suivait dans tous ses déplacements.

Son cousin de la branche aînée des Brienne, Gautier, duc d'Athènes, n'apparaît qu'une seule fois dans les retenues, tout comme Édouard de Beaujeu. Il était présent à la campagne de 1338, donc au début du conflit. On a vu plus haut que ces grands nobles servaient également le roi, peut-être en même temps mais sur un autre lieu de conflit. En effet, le duc d'Athènes fut par la suite chargé, comme son cousin, de diverses missions de commandement, notamment dans le nord du royaume, sur le front principal de la lutte. Son absence dans la compagnie du connétable n'induit pas qu'il se soit éloigné des combats, au contraire, puisqu'il devint, en son temps, connétable de France. Par extension, il est possible d'inscrire dans ce lien familial tous les hommes qui étaient attachés de près ou de loin à la maison de Brienne, ou aux familles qui lui étaient alliées comme les Châtillon. On peut ainsi retenir Édouard de Beaujeu, même s'il ne participa qu'à une seule campagne avec Raoul d'Eu.

Mais force est de constater que l'ensemble de ces chevaliers n'est pas uni au comte d'Eu par un lien de nature unique. Une solidarité en recouvre la plupart du temps une autre, voire deux, ou bien elle est tellement mêlée aux autres qu'il devient difficile de les distinguer. En ce sens, l'ensemble de ces relations complexes forme un écheveau d'une densité particulière et qui éclaire toute la complexité des réseaux de familiers.

\section{Le service de l'hôtel et la complexité de ces liens}

Après avoir défini, présenté et analysé de façon analytique les diverses solidarités s'exerçant dans les armées, il nous faut nuancer le propos dans la mesure où, dès le début de l'étude prosopographique, une certitude s'imposa : les liens qui unissaient ces hommes à leur chef étaient beaucoup plus complexes qu'il n'y paraissait. Les familiers du connétable furent rarement liés à lui par une seule forme de solidarité. D'une manière générale, elles étaient enchevêtrées les unes aux autres au point de former un écheveau serré de fraternité d'armes et de fidélité, d'intérêt et d'échange de service. Ils étaient ainsi des vassaux et des membres de l'hôtel du connétable, des voisins doublés de familiers intimes, à la fois parents et voisins comme Édouard de Beaujeu, etc. Chaque nature de lien se superposait donc à une ou 
plusieurs autres en même temps, ce qui intensifia et renforça les attaches existant entre ces hommes, mais en augmentant peut-être d'autant la situation de dépendance de certains vis-à-vis de leur patron. De plus, par le jeu des alliances matrimoniales de l'ensemble des grandes familles du royaume, tout ce réseau de nobles chevaliers était peu ou prou lié, ils étaient souvent cousins à des degrés plus ou moins proches : par exemple, le duc d'Athènes et Édouard de Beaujeu étaient cousins germains et avaient le même grand-père, le défunt connétable Gaucher de Châtillon. De même, Édouard de Beaujeu était un cousin éloigné du comte d'Eu. Pareillement, une communauté d'intérêts rapprochait ces barons, normands pour la plupart.

Mais il est un lien hiérarchique d'une autre nature qui vient dominer les autres : le service de l'hôtel. Dans l'entourage du connétable ainsi que dans sa bataille, se trouvaient en grande majorité des gens de son hôtel, officiers ou serviteurs. Nous rencontrons ainsi ses maîtres d'hôtel: Robert de Houdetot, Robert de La Heuse, Guillaume de Beuzeville et Jean Waliquet ${ }^{7}$, Pierre du Fay et Jean de Beuzeville ${ }^{8}$, son barbier Jacquinot, ou encore ses ménestrels. Le comte se déplaçait également avec des hommes directement impliqués dans le métier des armes comme des heaumiers et des maréchaux-ferrants, ou encore des armuriers. Le service de l'hôtel est cependant très difficile à repérer, voire impossible dans certains cas. En effet, cette mention est très rarement indiquée dans le registre de dettes du comte; il faut donc se reporter aux retenues qui se partagent en deux types. Cette distinction, dans la mesure où elle est toujours indiquée précisément, revêt par conséquent une importance particulière : les retenues du connétable concernent les «gens d'armes de l'hostel» ou les «gens d'armes» tout simplement. Il est significatif que le comte retienne «de son mesnage » les deux chevaliers du comté de Bar, Ferry de Chardogne et Louis de Corbon ${ }^{9}$. Le service domestique vient renforcer les liens déjà existants de covassalité et de compagnonnage d'armes.

Une exception existe au demeurant: la longue campagne de l'année 1340, qui vit sans aucun doute le plus grand rassemblement de troupes de la première partie de la guerre de Cent Ans, parmi les hommes du connétable comme parmi l'ensemble des combattants: la retenue du comte d'Eu concerne les «gens d'armes de l'hostel comme les gens d'armes en dehors $»^{10}$. Tous les combattants qui servaient le connétable étaient donc rassemblés en une seule retenue, la plus importante numériquement. Cette conjonction des familiers de l'hôtel et de familiers d'armes en un même service est un bon indice de la dimension qu'a revêtue pour les contemporains cette campagne de 1340. Son enjeu avait été bien saisi et c'est pour cette raison que la mobilisation fut aussi forte.

La retenue à l'hôtel du comte était un service mouvant, dont le renouvellement était fréquent, semble-t-il. Tel chevalier figure dans la retenue des gens d'armes de l'hôtel pour telle campagne et pas pour telle autre. Étant donné qu'une cinquantaine de personnes servait le connétable, dans sa maison comme aux armées, il semble difficile, malgré son train, qu'il ait pu entretenir un nombre aussi important d'officiers et serviteurs pendant plus de cinq ans. Le service de l'hôtel était donc tournant et l'on y retrouve tantôt l'un tantôt l'autre de ces hommes. Il est tout de

\footnotetext{
${ }^{7}$ B.n.F., n.a.f. 7413 , f. 172.

${ }^{8}$ En 1338, B.n.F., n.a.f. 7413 , f. $246 v^{\circ}$, et en 1340 , B.n.F., fr. 32510 , f. $176 v^{\circ}$.

${ }^{9}$ A.N., JJ 269 , f. $32 v^{\circ}$ et $33 r^{\circ}$.

${ }^{10}$ B.n.F., fr. 32510 , f. $175 r^{\circ}$ et fr. 9238 , f. 127-147.
} 
même possible de dégager un groupe de fidèles qui servit assidûment à la fois dans l'hôtel et dans les armées; ce sont indiscutablement les hommes les plus proches : Robert de Houdetot, Jean de Cayeu, les trois frères de la Heuse, Colin et Jean Doublel ou les cinq membres de la famille de Hotot. Certains chevaliers, vassaux du comte d'Eu, comme Baudouin de Balinghem ou Anguenier de Sainte-Beuve, ajoutent au lien vassalique ce lien hiérarchique : ils sont mentionnés comme étant de son hôtel à plusieurs reprises dans les campagnes auxquelles ils participèrent. Ils consolidèrent ainsi leur lien de vassalité par le service domestique auprès de leur seigneur.

Que ce soit au sein de l'hôtel ou dans les retenues du comte, il est par ailleurs possible de rapprocher les membres d'une même famille. Prenons l'exemple des Hotot, qui furent toujours de fidèles serviteurs de l'hôtel. Guillaume était panetier du comte pendant la campagne de Languedoc en été 1337, Jean participa à la semonce de Compiègne du mois de septembre 1339, comme Colart, qui servait alors sous Pierre de Bailleul, et comme Robert, qui était son porte-bannière. Le même principe vaut pour la famille de Beuzeville: Guillaume et Jean étaient maîtres d'hôtel du comte, ou encore pour celle de La Heuse : les trois frères, Guillaume, Jean et Robert, servirent assidûment le comte dans son hôtel et aux armées. Nous relevons enfin, dans les retenues du connétable, deux membres de la famille de Marigny, Robert et Thomas, parents du défunt Enguerrand. Il existait indubitablement une tradition de service du comte d'Eu dans certaines familles, comme si tous ces nobles normands s'étaient retrouvés en lui et en ses actions, comme s'il les avait rassemblés et avait incarné leurs aspirations. L'on pense alors au traité d'assistance conclu entre le roi Philippe VI et les barons normands, à la tête desquels figuraient notre comte d'Eu. Dans la liste des signataires se retrouvent plusieurs familiers du comte, comme le sire de Préaux, Guillaume de Beuzeville, Pierre, sire de Bailleul, Robert, sire de Houdetot, Robert de La Heuse et Colart de Hotot, sire d'Englesqueville.

On voit ainsi que non seulement le comte d'Eu avait réussi à attirer de nombreuses personnes à son service, au point de constituer un véritable réseau de familiers, mais qu'en outre, des rapprochements, voire des liens d'amitié s'étaient établis à l'intérieur même de ce réseau entre ses différents éléments. Les amis se retrouvèrent après le décès de leur patron et c'est à la mort du fils de ce dernier, le second connétable d'Eu, qu'intervinrent la rupture et la disparition du réseau, par le choix du prince auprès duquel ils s'engagèrent: qui au service du roi de Navarre, qui à celui du duc de Normandie.

Évoquons à grands traits l'existence de ce que l'on pourrait appeler un lien vassalique en abîme. Nous savons que Jean de Cayeu était un familier intime du comte d'Eu et qu'il servait dans sa retenue et dans son hôtel. Jean de Dargnies servait également sous le connétable mais il était plus précisément sous le gouvernement du sire de Senarpont en Flandre en 1338. Peut-on en conclure que les familiers des familiers du comte devenaient ses familiers ? Jean de Dargnies semble cependant plus directement lié au comte de Guînes, le fils du conétable, dont il était un compagnon. En ce sens, nous pensons qu'un lien de fraternité d'armes se superposait aux autres solidarités et venait les renforcer. Le connétable avait tout d'abord recruté ses hommes d'armes dans ses terres et dans sa famille, et il semblerait que l'habitude du combat, la guerre et les nombreuses campagnes menées ensemble (notamment le difficile siège de Tournai en été 1340) eussent renforcé les liens préexistants d'une fraternité incontournable dans le métier des armes. S'il existait dans 
ce cercle des liens d'amitié, ils se créèrent probablement à l'occasion des combats et du danger partagé. Ces chevaliers étaient sans doute voisins au départ, mais ils se connaissaient peu ou mal avant de se côtoyer quotidiennement dans la retenue du connétable. Il existe enfin un lien que nous n'avons pas encore mentionné et qui, encore une fois, se superpose aux précédents : la relation de l'employeur à ses employés, en l'occurrence à ses soudoyers, ses «retainers »: le connétable retenait ses hommes d'armes dans sa compagnie et payait leurs gages. Son registre de comptes regorge de paiements faits à ses chevaliers pour les diverses campagnes menées ensemble.

À travers cette analyse des liens unissant des chevaliers au sein d'un réseau et autour d'un patron, on remarque d'emblée l'importance du lien et combien les événements, les hommes, les décisions qu'ils prirent et les actions qu'ils engagèrent, les partis (politique ou autre) étaient enchevêtrés de manière inextricable. Cette conjonction des gens et des choses est encore plus sensible dans la destinée des hommes du connétable, et prolongea l'existence du réseau du comte d'Eu bien après sa disparition.

Quelle que soit la nature des liens ayant existé entre le comte et ses familiers, il apparaît clairement que les carrières de ces derniers ont, dans l'ensemble, suivi le même cheminement, et qu'elles ont toutes réellement commencé après la mort des deux connétables d'Eu, soit vers 1350. En schématisant quelque peu, deux périodes se dégagent de l'ensemble des destinées de ces chevaliers: la première au service des deux connétables d'Eu (1337-1350), la deuxième soit auprès du jeune roi de Navarre, Charles, soit auprès du dauphin Charles, régent du royaume et nouveau duc de Normandie, qui devint dès lors leur seigneur naturel et autour duquel ils firent cercle.

Concernant l'ensemble des chevaliers, l'impression dominante au regard de ces années passées dans l'entourage des deux connétables d'Eu est la continuité : en effet, après avoir étudié les deux retenues dont nous disposons pour les campagnes du comte d'Eu et de Guînes en 1346, nous pouvons conclure que les hommes du père servirent le fils. Une douzaine d'entre eux suivit le second connétable d'Eu au siège d'Aiguillon puis en Normandie, à Harfleur et Caen, où le comte fut pris par les Anglais ${ }^{11}$. Nous retrouvons ainsi Jean de Bailleul, dit Gauvain, Baudouin et Tassart de Balinghem, qui était depuis devenu chevalier, Jean de Bauchain, Guillaume de Beuzeville, Louis de Courbon, Jean et Colin Doublel, Friquet de Fricamps, Eudes de Grancey, Dreu de Mello, Fremaut d'Oust et Robert Le Thiois. Nous retrouverons ensuite plusieurs de ces hommes auprès du roi de Navarre ou du dauphin Charles. En 1350, après l'exécution sommaire du second connétable d'Eu, tous ses proches famille, amis, clients, compagnons d'armes, serviteurs - se muèrent en un vaste réseau de mécontents qui ébranla le pouvoir royal déjà déstabilisé par les luttes de pouvoir et d'influence. Les fidèles des comtes d'Eu nourrirent dès lors de nombreux griefs envers leur nouveau roi et il n'est pas étonnant qu'une poignée d'entre eux ait choisi le camp de l'opposition en la personne du roi de Navarre. Par exemple,

${ }^{11}$ B.n.F., fr. 32510, f. $185 r^{\circ}-187 r^{\circ}$ et $188 r^{\circ}$. 
«Colin Doublel et Jean de Fricamps croiront venger le comte d'Eu en assassinant Charles d'Espagne $»^{12}$.

Après 1350 , l'unité du réseau se rompit et deux principaux schémas de carrière s'imposèrent rapidement aux anciens clients et compagnons des comtes d'Eu : le service du roi et de son fils aîné, le dauphin Charles, qui devint duc de Normandie en $1355^{13}$, ou celui du roi de Navarre, avec les fidélités qui en découlaient. Dans les deux cas, ce fut toute la noblesse du nord-ouest du royaume, principalement normande et picarde, qui se rangea dans l'un ou l'autre camp derrière ces princes des fleurs de lis, au service d'un homme pour lequel les intérêts normands comptaient, qu'il s'agisse de Charles le Mauvais ou du dauphin.

Malgré l'influence exercée par le roi de Navarre dans le duché de Normandie, la plupart des compagnons normands des connétables d'Eu se rangèrent auprès de leur seigneur naturel, le duc Charles, mais les chevaliers picards et bourguignons aussi, comme s'il était logique qu'ils se tournent vers le roi et/ou son héritier après avoir servi dans l'entourage du connétable de France: Robert de Houdetot devint ainsi maître des arbalétriers en 1350 et capitaine général au duché, il fut de plus largement impliqué dans les États de Normandie de 1355. Anguenier de SainteBeuve fut commissaire royal pour le subside des gens de guerres aux même États de 1355 et servit dans l'armée du dauphin. Baudouin de Balinghem resta pour sa part dans le nord du royaume pour y garder des places ; on peut donc le ranger aux côtés du dauphin. Tassart de Balinghem était sergent d'armes du roi et châtelain de Niort en 1350. Jean de Bauchain mourut au siège de Saint-Valéry-sur-Somme en 1358, en combattant aux côtés du connétable Moreau de Fiennes. Guillaume de Beuzeville était capitaine de diverses places en Normandie pour le compte du duc. Jean de Beuzeville servit en 1356 sous Amaury de Meulan, lieutenant du duc. Ferry de Chardogne était maréchal de l'ost du duc dès 1345 et Jean de La Heuse, dit le Baudrain, son maréchal en 1355, puis son chambellan et enfin amiral de France à partir de 1359 . Il resta par la suite au service de ce prince lorsqu'il régna après 1364 . Jean de Bailleul reçut plusieurs dons du roi et du duc de Normandie en 1362 et 1363. Quant à Édouard de Beaujeu et au duc d'Athènes, ils devinrent respectivement maréchal de France en 1347 et connétable en 1356. Geoffroy de Charny était indiscutablement un proche du roi Jean, comme Eudes de Grancey et Enguerrand Quiéret, qui devint amiral de France en 1357. Lors de la création de l'ordre de l'Étoile et de sa première fête en janvier 1352, le sire de Balinghem et Geoffroy de Charny étaient présents $^{14}$, ce qui induit qu'ils étaient membres de cet ordre de chevalerie. La Chronique des quatre premiers Valois ${ }^{15}$ montre bien que Robert de Houdetot et le Galois de La Heuse, tous deux titulaires de charges officielles importantes, étaient des fidèles du roi et de son fils et qu'ils suivirent le duc de Normandie, qui était aussi leur seigneur naturel. Il en va de même pour Geoffroy de Charny et le duc d'Athènes. On voit par cette énumération que la grande majorité des familiers des comtes d'Eu demeurèrent des fidèles de la cause royale.

${ }^{12}$ R. Cazelles, La société politique et la crise de la royauté sous Philippe VI de Valois, Paris, 1958, p. 252.

${ }^{13}$ F. Autrand, Charles V le Sage, Paris, 1994, p. 165.

${ }^{14}$ L. Pannier, La noble maison de Saint-Ouen, la Villa Clippiacum et l'ordre de l'Étoile, Paris, 1872, p. 95

${ }^{15}$ Éd. S. Luce, Paris, 1862, p. 37-41. 
Quant aux proches du roi de Navarre issus de la clientèle des comtes d'Eu, ils étaient exclusivement normands. Nous retrouvons dans son entourage Colin Doublel et Friquet de Fricamps, Pierre de Préaux et les membres de la famille de Hotot ${ }^{16}$. Ils se tournèrent vers lui de manière naturelle en pensant s'être trouvé un chef qui avait cristallisé leurs griefs et qui était capable d'incarner leurs aspirations et leur désir de vengeance. Mais les fidélités à ce jeune prince se recrutèrent beaucoup plus largement, jusque dans la Picardie ${ }^{17}$, le nord du royaume et même l'Auvergne ${ }^{18}$. Il sut tirer parti du mécontentement des gens du nord et susciter des allégeances nombreuses au sein d'une noblesse récalcitrante du fait du supplice du second connétable d'Eu mais également parce que le roi avait disposé de ses biens en dehors d'elle, au grand bénéfice de Charles d'Espagne et des enfants de Robert d'Artois. Cela engendra de nombreuses adhésions à la cause du roi de Navarre, qui se sentit, grâce à ces nouveaux partisans, conforté dans ses revendications et ses complots.

Ainsi, l'étude de l'entourage du comte d'Eu a mis en lumière les modes de recrutement qu'il utilisait volontiers pour former sa compagnie, en premier lieu le lien régional. Cependant, d'autres rapports existaient au sein de ce réseau, complexes et connexes à la fois. Le service à l'hôtel du comte, un certain compagnonnage d'armes, le partage de la vassalité, toutes ces relations d'homme à homme ont permis au comte d'Eu de se constituer un véritable réseau de clients et de familiers, voir d'amis, et ce qu'il s'agisse de voisins, de parents, de vassaux ou de serviteurs. Au terme de cette synthèse, il apparaît que ces chevaliers ne figurent plus dans les sources après 1360 , à quelques exceptions près. Cette date représente donc une charnière et correspond à un changement de génération de combattants. Ces hommes, qui prirent leur essor sous l'aile du connétable d'Eu et qui ont parfois constitué les cadres dirigeants de l'armée de la fin du règne de Philippe VI, ont incarné la première génération des combattants de la guerre de Cent ans. Ils laissèrent, après 1360, la place à une relève plus jeune, représentée par la génération de Bertrand du Guesclin.

Émilie Lebailly Archives départementales d'Eure-et-Loir

\footnotetext{
${ }^{16}$ R. Cazelles, «Le parti navarrais jusqu'à la mort d'Étienne Marcel », Bulletin Philologique et Historique du Comité des travaux historiques et scientifiques, 1960, p. 845-846.

${ }^{17}$ Notamment dans la vidamie d'Amiens, appartenant à Marguerite de Picquigny, cousine des défunts connétables. Elle embrassa très probablement les sympathies de leur clientèle (R. Cazelles, «Le parti navarrais... », op. cit., p. 856 et A.N., JJ 269, f. 80r ${ }^{\circ}$ ).

${ }^{18}$ R. Cazelles, «Le parti navarrais... », op. cit., p. 847.
} 